\title{
Biochemistry and Cell Biology
}

\section{Isovitexin attenuates tumor growth in human colon cancer cells through the modulation of apoptosis and epithelial- mesenchymal transition via PI3K/Akt/mTOR signaling pathway}

\begin{tabular}{|r|l|}
\hline Journal: & Biochemistry and Cell Biology \\
\hline Manuscript ID & bcb-2021-0045.R3 \\
\hline Manuscript Type: & Article \\
\hline Aute Submitted by the \\
Complete List of Authors: & $\begin{array}{l}\text { 04-Jun-2021 } \\
\text { Zhu, Hao; Yantaishan Hospital, Nantaishan Hospital } \\
\text { Jiang, Maozhu; Yantaishan Hospital }\end{array}$ \\
\hline Keyword: & $\begin{array}{l}\text { colon cancer cells, cell proliferation, cell apoptosis, EMT, tumor bearing } \\
\text { mice }\end{array}$ \\
\hline $\begin{array}{r}\text { Is the invited manuscript for } \\
\text { consideration in a Special } \\
\text { Issue? : }\end{array}$ & Not applicable (regular submission) \\
\hline &
\end{tabular}

\section{SCHOLARONE ${ }^{m}$ Manuscripts}


1 Isovitexin attenuates tumor growth in human colon cancer cells through the modulation of

2 apoptosis and epithelial-mesenchymal transition via PI3K/Akt/mTOR signaling pathway

$3 \quad \mathrm{Hao} \mathrm{Zhu}^{1 *}$, Na Zhao ${ }^{1}$, Maozhu Jiang ${ }^{2}$

$4 \quad{ }^{1}$ Department of Gastroenterology, Yantaishan Hospital, Yantai, 264003, Shandong, China.

$5 \quad 2$ Department of Radiotherapy, Yantaishan Hospital, Yantai, 264003, Shandong, China.

$6{ }^{*}$ Corresponding author,

7 Hao Zhu,

8 Department of Gastroenterology, Yantaishan Hospital, No.10087, Keji Avenue, Laishan District,

$9 \quad$ Yantai, Shandong, China.

10 Email: zhuhao0729@163.com

11 Tel: +86-13963895391

12 Running Title: Isovitexin inhibits colon cancer 


\section{Abstract}

Isovitexin, a biologically active flavone C-glycosylated derivative, has a variety of biological activities. We aimed to identify the effect of isovitexin (Isov) on colon cancer. The human colonic epithelial cell (HCEC), and cancer cells were treated with Isov, Cell Counting Kit-8 (CCK8) was used to detect cell proliferation and calculate half-inhibitory concentration $\left(\mathrm{IC}_{50}\right)$. The biological activity of cancer cells were assessed. The tumor size and volume were recorded. The expression levels of proteins were analyzed by western blot. Isov inhibited cancer cells proliferation, while had little cytotoxicity on HCEC. Isov significantly attenuated cell proliferation, migration, invasion and epithelial-mesenchymal transition (EMT), induced cell apoptosis, and that trends were blocked by insulin-like growth factor-1 (IGF-1) treated. The expression levels of phosphorylated phosphatidylinositol 3-kinasep (p-PI3K), phosphorylated protein kinase B (p-Akt), phosphorylated mammalian target of rapamycin (p-mTOR), and B-cell lymphoma-2 (Bcl-2) evidently decreased when treated with Isov, while the levels of Bcl2-associated X (Bax), and caspase-3 significantly increased. After Isov treatment, the tumor volume and weight were decreased, the levels of p-PI3K, p-Akt, p-mTOR, and Bcl-2 significantly decreased in tumor tissues. Our finding demonstrated that Isov could inhibit cancer cells migration, invasion and EMT. Isov maybe a new potentially treatment medicine for colon cancer.

Keywords: colon cancer cells; cell proliferation; cell apoptosis; EMT; tumor bearing mice

\section{Introduction}

Colon cancer is the third most common cancer and the second most deadly (Rademaker et al, 2019). It is one of the common clinical malignancies. In 2018, there were approximately 1.1 
million new cases worldwide and more than 550,000 deaths (Bray et al, 2018). In China, colon cancer is also the most common gastrointestinal malignant tumor (Fu et al, 2019). With the continuous improvement of people's living standards, the incidence of colon cancer has shown an upward trend year by year. The symptoms of colon cancer are insidious in the early stage. As the tumor grows, symptoms such as changes in bowel habits, blood in the stool, diarrhea, constipation, and local abdominal pain are manifested. In the late stage, symptoms such as anemia and weight loss are manifested (McDermott et al, 1981; Phipps et al, 2008). Due to the difficulty in early diagnosis of colon cancer, most patients develop clinical symptoms to the middle and late stages when they seek medical attention, and most patients have metastasized. The effect of supplementary radiotherapy and chemotherapy after surgery is not good, it seriously endangers peoples' health (Sun et al, 2012). It is necessary to find effective drugs to inhibit colon cancer with small side effects.

Isovitexin (apigenin 6-C-glucoside) is a natural flavonoid compound, usually purified together with vitexin. It is an isomer of vitexin and has a variety of biological activities. Isovitexin (Isov) is active ingredient of many traditional Chinese medicines and exists in various medicinal plants, such as the fruit of the cucurbitaceae fruit or pigeon peas, wheat, triangle fruit and bamboo leaves (He et al, 2016). Isov has multiple pharmacological activities, such as antioxidant, anti-inflammatory, antibacterial, anti-Alzheimer's disease (Lin et al, 2005; Zhang et al, 2011; Choi et al, 2014; $\mathrm{Mu}$ et al, 2018). Furthermore, there are studies about Isov has anti-cancer effects (Ganesan and Xu, 2017; Hanafi et al, 2017; Lv and Qiao, 2018; Liang et al, 2019). However, it is unknown whether Isov has efficacy on colon cancer, and how it represses proliferation and epithelial-mesenchymal transition (EMT) of colon cancer. In addition, the 
5-year survival rate of colon cancer is low, the recurrence rate, and metastasis rate are high (Allemani et al, 2018). Traditional Chinese medicine has a significant effect on colon cancer, especially in the prevention of recurrence and metastasis in the late stage of colon cancer. This study aimed to evaluate the proliferation inhibitory activity of Isov in colon cancer.

\section{Materials and methods}

\section{Cell lines and groups}

Human colonic epithelial cell (HCEC), and cancer cells HT-29, SW480, HCT116, LoVo (ATCC, USA) were incubated in Roswell park memorial institute (RPMI, Solarbio, Beijing, China) 1640 medium with $10 \%$ fetal bovine serum (FBS, Hyclone, USA). Cancer cells were fostered at $37^{\circ} \mathrm{C}$ with $5 \% \mathrm{CO}_{2}$ until they reached $80 \%$ confluence. Cells were collected and resuspend, adjusted the cell concentration, $5 \times 10^{3}$ cells were cultured in 96 -well plates. After incubation at $37^{\circ} \mathrm{C}$ with $5 \% \mathrm{CO}_{2}$ for $24 \mathrm{~h}$, the cells were treated with the indicated concentrations of Isov $(0,2.5,5,10$, 20, 40, $80 \mu \mathrm{mol} / \mathrm{L}$ ). Cell Counting Kit-8 (CCK-8, Beyotime, China) was used to measure cell proliferation. The absorbance was measured at $450 \mathrm{~nm}$. The cells growth inhibition rate and $\mathrm{IC}_{50}$ were calculated.

The cells were randomly divided into four groups: control, Isov, insulin-like growth factor-1 (IGF-1), and Isov + IGF-1 groups. The recombinant human IGF-1 (10 nM, Sigma-Aldrich, USA) and Isov (60 $\mu \mathrm{mol} / \mathrm{L}$, Thermo Scientific, Shanghai, China) were treated with cells, respectively.

\section{Scratch wound healing assay}

Cells were digested with $0.25 \%$ trypsin- Ethylene Diamine Tetraacetic Acid (EDTA) solution (Sigma, USA), centrifuged $(350 \times \mathrm{g}, 5 \mathrm{~min})$, the pellets were collected and re-suspended to $3 \times$ $10^{5}$ cells $/ \mathrm{mL}$. $1 \mathrm{~mL}$ of cell suspension was added to 6-well plate and incubated overnight. Then, 
HCT116 and LoVo cells were treated with Isov or IGF-1, and $24 \mathrm{~h}$ after creating the wound. Scratch was performed using a $10 \mu \mathrm{L}$ pipette tip. The cell migration photos were taken for analysis.

\section{Transwell assay}

Cells were collected using a $0.25 \%$ trypsin-EDTA solution, the medium was removed and the cells were resuspended in serum-free RPMI 1640 at a cell density of $2 \times 10^{5} / \mathrm{mL}$. $50 \mu \mathrm{L}$ Matrigel (BD Bioscience, USA) was added on the membrane of the upper chamber, solidified at $37^{\circ} \mathrm{C}$ for 30 min. Complete medium $(600 \mu \mathrm{L})$ containing $10 \%$ FBS was added to a $24-$ well plate and 100 $\mu \mathrm{L}$ cell suspension was seeded on the membrane, incubated at $37^{\circ} \mathrm{C}, 5 \% \mathrm{CO}_{2}$. Transwell chambers were collected after $24 \mathrm{~h}$ incubation, then fixed in cold methanol for $30 \mathrm{~min}$. Cells were stained with $0.5 \%$ crystal violet at room temperature for $20 \mathrm{~min}$. The number of invasion cells were observed under an optical microscope (Olympus, Japan). Nine bright fields were randomly selected for photographing to calculate the number of invading cells on the membrane.

\section{Clone formation assay}

HCT116 and LoVo cells were vaccinated in a 24 -well plate at a density of 500 cells/well. After 12 days of different administration, the cells were stained with $1 \%$ methyl rosamine chloride and the number of visible colonies were counted. The relative cloning ability is calculated as relative cloning ability $=$ (average number of clones in treated groups / average number of clones in control group) $\times 100 \%$.

\section{Flow cytometry assay}

After $24 \mathrm{~h}$ treatment, trypsin was used to digeste (centrifugation, $1500 \times \mathrm{g}, 5 \mathrm{~min}$ ) and suspension cells. Annexin V-FITC/PI kit (Solarbio, Beijing, China) was used to analyze cells 
apoptosis. Cells were incubated with Annexin V-FITC and propidium iodide (PI) for $20 \mathrm{~min}$ at room temperature in dark, and detected by the flow cytometer (Beckman Coulter Inc., USA).

\section{Colon cancer xenograft tumor model}

A total of 18 BALC/nude mice (female, 4-6 weeks of age, Jinan Peng Yue experimental animal breeding Co., Ltd., Shandong, China) were maintained at $20 \sim 26^{\circ} \mathrm{C}$, daily temperature $\leq 4^{\circ} \mathrm{C}$, humidity $50 \sim 60 \%, 12 \mathrm{~h}$ light/dark cycle. The mice were free access to water. HCT116 cells were cultured, the cells were grown in the logarithmic phase and mixed with serum-free RPMI 1640 to a final suspension of $2 \times 10^{6} / \mathrm{mL}$. The cell suspensions $(0.2 \mathrm{~mL})$ were injected subcutaneously on the right flank in nude mice. Animal experiments were carried out accordance with the recommendations of the National Institutes of Health Guidelines for the Nursing and Use of Laboratory Animals. (National Research Council (US) Institute for Laboratory Animal Research, 1996). All experiments were approved by the animal ethics committee of Yantaishan Hospital.

\section{Animal groups and administration}

After tumor volume reached $100 \mathrm{~mm}^{3}$, the mice were treated with Isov for 4 weeks. BALC/nude mice were randomly divided into 2 groups $(\mathrm{n}=9)$ : control, and Isov $(20 \mathrm{mg} / \mathrm{kg})$ groups. The Isov group received intragastric administration. Tumor growth were measured by a digital caliper. The tumor length (long diameter, a) and width (short diameter, b) of tumor growth were observed once a week. The tumor volume $(\mathrm{V})$ was calculated using the following formula: $\mathrm{V}=0.5 \times(\mathrm{a} \times$ $b^{2}$ ). The formula of tumor growth inhibition rate as follows: Tumor growth inhibition rate $=$ (average tumor volume in control group - average tumor volume in Isov group) / average tumor volume in control group $\times 100 \%$. The body weight of mice was recorded once a week. The mice were sacrificed and the tumor bodies were extracted, and weighed, then frozen for western blot analysis. 


\section{Western blot}

Added $800 \mu \mathrm{L}$ whole protein extract radioimmunoprecipitation lysate (RIPA, Solarbio, Beijing, China), containing $10 \%$ protease inhibitor phenylmethylsulfonyl fluoride (PMSF) to colon cancer cells or tumor tissues to extract protein. Protein concentration was determined by Bicinchonininc acid (BCA) kit (Thermo Fisher Scientific, USA) following manufacturer's introduction. Protein samples were loaded on $10 \%$ sodium dodecyl sulfate polyacrylamide gel electrophoresis (SDS-PAGE) then transferred onto Poly(vinylidene fluoride) membrane (PVDF, Merck, Darmstadt, Germany) at $80 \mathrm{~V}$ for $30 \mathrm{~min}$, blocked for $1 \mathrm{~h}$ with 5\% skimmed dry milk in Tris $\mathrm{HCl}$ buffer solution + Tween (TBST) solution. Antibodies were diluted with $3 \%$ bovine serum albumin in TBST. Antibodies included: Bcl-2 (1:50, MA5-11757, Invitrogen, USA), Bax (1:1000, 33-6400, Invitrogen, USA), Caspase-3 (1 $\mu \mathrm{g} / \mathrm{mL}$, MA5-11521, Invitrogen, USA), anti-phospho (p)-PI3Kp85 (1 : 2000, ab182651; Abcam, UK), PI3K (1:1000, Cell Signaling Technology, USA), p-Akt (Ser473, 1:1000, Cell Signaling Technology, USA), p-mTOR (1:1000, \#5536, Cell Signaling Technology, USA), E-cadherin (1:1000, Cell Signaling Technology), N-cadherin (1:1000, Cell Signaling Technology), vimentin (1:1000, Cell Signaling Technology), snail (1:1000, Cell Signaling Technology), slug (1:1000, Cell Signaling Technology), zona occludens 1(ZO-1, 1:500, ab216800; Abcam), ,incubated with horseradish peroxidase (HRP) goat anti-rabbit $\operatorname{IgG}(1: 2000$, Proteintech, USA) for $1 \mathrm{~h}$. The signals were detected by enhanced chemiluminescence (ECL) system. The levels of proteins were standardized with glyceraldehyde-3-phosphate dehydrogenase (GAPDH), the quantification of grayscale scanning was performed by Image $\mathrm{J}(\mathrm{NIH})$ software.

\section{Statistical analysis}


The data was analyzed by statistical program for social sciences (SPSS) statistical software. The results were showed as mean \pm standard deviation (SD). One-way analysis of variance (ANOVA) followed by least-significant difference (LSD) test was used to analyze the data among groups. Student's t-test was used to analysis the difference between groups. $P$ value less than 0.05 $(P<0.05)$ was considered significant difference.

\section{Results}

\section{Isov inhibits colon cancer cells viability}

The results were shown in Figure 1A, the sensitivity of four colon cancer cells to Isov were consistent. Isov inhibited colon cancer cell lines in a dose-dependent manner, with $\mathrm{IC}_{50}$ of HT29 cell was $37.24 \pm 2.56 \mu \mathrm{mol} / \mathrm{L}$, SW480 was $33.53 \pm 2.45 \mu \mathrm{mol} / \mathrm{L}$, HCT116 was $29.79 \pm 2.32$ $\mu \mathrm{mol} / \mathrm{L}$, and LoVo was $19.31 \pm 2.49 \mu \mathrm{mol} / \mathrm{L}$. The $\mathrm{IC}_{50}$ of $\mathrm{HCEC}$ was higher than $80 \mu \mathrm{mol} / \mathrm{L}$. The effect of Isov on HCEC has no obvious cytotoxicity. Then, we divided the cells into 4 groups and observed the effect of Isov on cell proliferation. The colony formation ability of colon cancer cells were decreased after Isov treatment, contrary to IGF-1 treatment. When the cells treated with both Isov and IGF-1, the colony formation ability was decreased compared to IGF-1 treatment alone (Figure 1B-D).

\section{Isov attenuates cells migration and invasion ability}

The effect of Isov on migration and invasion of colon cancer cells were assessed. We noted that administration of IGF-1 significantly promoted cell migration and invasion compared with cells in control group, while the abilities of invasion were blocked by Isov $(P<0.05)$. Especially, we observed that the wound healing was $100 \%$ in HCT-116 and LoVo cells because of IGF-1 (Figure 2A-D). Through the invasion assay, we observed that IGF-1 induced to a pronounced 
increment of invaded cells when compared with cells in control group $(P<0.01$, Figure 2E-F). Treatment with Isov blocked cell migration and invasion. When cells treated with both Isov and IGF-1, the cells migration and invasion ability were decreased compared to IGF-1 treatment alone.

\section{Isov induces cell apoptosis in colon cancer cells}

As presented in Figure 3, the cell apoptotsis rate in Isov group was significantly increased compared to control group, while cell apoptosis rate in IGF-1 group was decreased. After treatment with both Isov and IGF-1, the cell apoptosis rate was increased compared to IGF-1 treatment alone $(P<0.05)$. The result indicated that Isov could inhibited the promoting effect of IGF-1. In addition, the expression level of Bcl-2 decreased in Isov group, while the levels of Bax, and caspase-3 increased $(P<0.01)$. The results in IGF-1 group were opposite to those of Isov group. When the cells treated with both Isov and IGF-1, the levels of Bax, caspase-3 decreased compared with IGF-1 treatment alone $(P<0.01)$.

\section{Isov attenuates EMT in colon cancer cells}

EMT plays a crucial role in cancer cells migration and invasion (Findlay et al, 2014). We investigated the expression of EMT biomarker, whole cell extracts were probed for E-cadherin, N-cadherin, ZO-1, mesenchymal markers (vimentin), EMT-related transcription factor (slug), and snail expression (Figure 4). The western blot results showed that the protein expression of E-cadherin and ZO-1 were significantly higher in the Isov group than in the control, IGF-1, and Isov + IGF-1 group. IGF-1 elevated the N-cadherin, vimentin, slug, and snail on the protein level, and Isov alone or in co-treatment with IGF-1 significantly diminished N-cadherin, vimentin, slug, and snail levels and increased E-cadherin, and ZO-1expression $(P<0.01)$. 


\section{Isov inhibites the activation of PI3K/Akt/mTOR signaling}

The expression level of p-PI3K, p-Akt, and p-mTOR in Isov group significantly decreased compared with control group, while the proteins levels increased in IGF-1 group $(P<0.01$, Figure 5). IGF-1 is an activator of PI3K/Akt signaling pathway. When the cells treated with both Isov and IGF-1, the proteins levels were decreased compared with IGF-1 treatment alone. The results revealed that the inhibitory effect of Isov on colon cancer cells maybe relate to PI3K/Akt/mTOR signaling pathway.

\section{Isov inhibits tumor growth in mice}

In order to verify the cell experiment, we established a xenograft tumor model in nude mice by HCT116 cells. As presented in Figure 6, the tumor size and shape were observed directly. We found that the tumor weight and volume were lower in Isov treated mice compared with those in control group $(P<0.05)$. In addition, the tumor inhibition rate in Isov group was $41.74 \%$. The results indicated that Isov inhibited the tumor growth.

\section{Isov attenuates EMT in vivo}

To verify the change of EMT in xenograft, we investigated the expression of EMT biomarker in transplanted tumor tissue. Western blot results presented that Isov treatment significantly decreased N-cadherin, vimentin, slug, and snail levels and increased E-cadherin, and ZO-1expression (Figure 7, $P<0.05$ ). The results were consistent with in vitro.

\section{Isov inhibites the activation of PI3K/Akt/mTOR signaling and induces cell apoptosis in vivo}

The expression of $\mathrm{p}-\mathrm{PI} 3 \mathrm{~K}, \mathrm{p}-\mathrm{Akt}$, and $\mathrm{p}-\mathrm{mTOR}$ proteins in tumor tissues were contrasted in Figure 8. The results were consistently with those in vitro. The expression levels of PI3K, p-Akt, and $\mathrm{p}$-mTOR proteins were limited in tissues of Isov group $(P<0.05)$. The expression levels of 
212 p-PI3K, p-Akt, p-mTOR, and Bcl-2 protein significantly decreased in tumor tissues of Isov group than those in control group while the levels of Bax and caspase- 3 increased $(P<0.05)$. The results suggested that Isov inhibited the activation of PI3K/Akt/mTOR signaling and induced tumor cell apoptosis in vivo.

\section{Discussion}

Previous studies have demonstrated that deregulation of cancer cell proliferation and apoptosis is 
formation assay, we found that Isov could effectively inhibited colon cancer cells migration, invasion, and proliferation. IGF-1 treatment significantly enhanced the colon cancer cells activity. When the cells treated with both Isov and IGF-1, the activity was significantly inhibited compared to IGF-1 treatment alone.

PI3K/Akt/mTOR pathway is overactive in many cancers, it is an important part of colon carcinogenesis. In addition, it plays an important role in acquiring drug resistance and metastasis. Some natural and synthetic small molecules have been found to inhibit colon cancer effectively through down-regulation PI3K/Akt/mTOR pathway (Narayanankutty, 2019; Zhu et al, 2020). IGF-1, as an activator of PI3K/Akt signaling pathway, we detected the expression levels relative protein relate to $\mathrm{PI} 3 \mathrm{~K} / \mathrm{Akt} / \mathrm{mTOR}$ pathway, the results indicated that Isov treatment could restraint of PI3K/Akt/mTOR pathway activation. In addition, Isov also restraint of PI3K/Akt/mTOR pathway activation induced by IGF-1.

The abnormal activity of PI3K/Akt/mTOR in a variety of cancers can promote cell proliferation and inhibit cell apoptosis, result in the aggravation of the disease course (Costa et al, 2018). Apoptosis is a highly complex and strict process of programmed cell death. It is a physiological mechanism of cell death regulated by pro-apoptotic and inhibitory factors. Bcl-2 and Bax play a critical role in the regulation of apoptosis. Bax promotes apoptosis, while Bcl-2 has the opposite effect (Dlugosz et al, 2006). Therefore, we detected the efficacy of Isov on colon cancer cell apoptosis. From flow cytometry, we found that Isov could induced cells apoptosis, while cells apoptosis in IGF-1 group was decreased. When the cells treated with both Isov and IGF-1, the cell apoptosis rate was increased compared to IGF-1 treatment alone. Furthermore, the expression level of Bcl-2 downregulation while the expression levels of Bax and caspase-3 
upregulation after Isov treatment. All of the results indicated that Isov could effectively attenuated colon cancer in vitro. In addition, we investigated the effect of Isov on tumor- bearing nude mice. Compared with the control group, Isov effectively inhibited tumor growth, tumor volumes were significantly smaller than that in the control group. The expression levels of Bcl-2 protein and $\mathrm{PI} 3 \mathrm{~K} / \mathrm{Akt} / \mathrm{mTOR}$ pathway related protein decreased, while Bax and caspase-3 increased. Our study results are consistent with these studies (Hanafi et al, 2017; da Silva et al, 2018; Lv and Qiao, 2018; Liang et al, 2019). Isov could effectively promotes cancer cells apoptosis. Furthermore, PI3K/Akt/mTOR signaling pathway plays an important role in cancer process, includes promote EMT and angiogenesis (Lee et al, 2019; Li et al, 2019). We investigated EMT biomarkers expression in vitro and in vivo. The results presented that Isov could inhibited EMT. Previous study indicated that the inhibition of PI3K/Akt/mTOR signaling pathway can suppress the EMT in colorectal cancer (Lee et al, 2019; Wei et al, 2019). Our study suggested that Isov could reversed the activation of PI3K/Akt/mTOR pathway induced by IGF-1, and dinminished EMT, the results were consistent with previous studies (Lee et al, 2019; Wei et al, 2019). However, our study only investigate the effect of Isov on PI3K/Akt/mTOR signaling pathway. Due to the complexity of the body's response, its mechanism of action may be through a variety of pathways. In future studies, it is necessary to evaluate other biological functions of Isov.

\section{Conclusion}

In summary, Isov inhibits colon cancer cells proliferation in vitro and in vivo, and promotes cell apoptosis. The effectively mechanism may be related to inhibit the expression of phosphorylated PI3K, phosphorylated Akt, and phosphorylated mTOR, the key factors of PI3K/Akt/mTOR 
signaling pathway, initiate apoptosis, and suppress EMT. Our study provides an evidence about Isov as a potential resister to colon cancer. However, the efficacy of Isov still needs a large number of clinical trials to verify.

\section{Acknowledgements}

Not applicable.

\section{Availability of data and materials}

Data collected or analyzed during this study are included in this article.

\section{Author contributions}

All authors contributed to the study conception and design. Material preparation, data collection, and analysis were performed by HZ, NZ and MJ. The first draft of the manuscript was written by HZ, all authors commented on previous versions of the manuscript. All authors read and approved the final manuscript.

\section{Conflict of interests}

The authors declared that there was no conflict of interest in the study.

\section{References}

Allemani C., Matsuda T., Di Carlo V., Harewood R., Matz M., Nikšić M., et al; CONCORD Working Group. 2018. Global surveillance of trends in cancer survival 2000-14 (CONCORD-3): analysis of individual records for 37513025 patients diagnosed with one of 18 cancers from 322 population-based registries in 71 countries. Lancet. 391(10125):1023-1075.

Banik K., Ranaware A.M., Deshpande V., Nalawade S.P., Padmavathi G., Bordoloi D., et al. 2019. Honokiol for cancer therapeutics: A traditional medicine that can modulate multiple oncogenic targets. Pharmacol Res. 144:192-209. 
Bray F., Ferlay J., Soerjomataram I., Siegel R.L., Torre L.A., Jemal A. 2018. Global cancer statistics 2018: GLOBOCAN estimates of incidence and mortality worldwide for 36 cancers in 185 countries. CA Cancer J Clin. 68(6):394-424.

Chatterjee A., Chattopadhyay D., Chakrabarti G. 2015. MiR-16 targets Bcl-2 in paclitaxel-resistant lung cancer cells and overexpression of miR-16 along with miR-17 causes unprecedented sensitivity by simultaneously modulating autophagy and apoptosis. Cell Signal. 27(2):189-203.

Choi J.S., Islam M.N., Ali M.Y., Kim E.J., Kim Y.M., Jung H.A. 2014. Effects of C-glycosylation on anti-diabetic, anti-Alzheimer's disease and anti-inflammatory potential of apigenin. Food Chem Toxicol. 64:27-33.

Costa R.L.B., Han H.S., Gradishar W.J. 2018. Targeting the PI3K/AKT/mTOR pathway in triple-negative breast cancer: a review. Breast Cancer Res Treat. 169(3):397-406.

da Silva I.C.V., Kaluđerović G.N., de Oliveira P.F., Guimarães D.O., Quaresma C.H., Porzel A., et al. 2018. Apoptosis caused by triterpenes and phytosterols and antioxidant activity of an enriched flavonoid extract from passiflora mucronata. Anticancer Agents Med Chem. 18(10):1405-1416.

Dlugosz P.J., Billen L.P., Annis M.G., Zhu W., Zhang Z., Lin J., et al. 2006. Bcl-2 changes conformation to inhibit Bax oligomerization. EMBO J. 25(11):2287-2296.

Findlay V.J., Wang C., Watson D.K., Camp E.R. 2014. Epithelial-to-mesenchymal transition and the cancer stem cell phenotype: insights from cancer biology with therapeutic implications for colorectal cancer. Cancer Gene Ther. 21(5):181-187.

Fu X., Huang Y., Fan X., Deng Y., Liu H., Zou H., et al. 2019. Demographic trends and 
KRAS/BRAFV600E mutations in colorectal cancer patients of South China: A single-site report. Int J Cancer. 144(9):2109-2117.

Ganesan K. and Xu B. 2017. Molecular targets of vitexin and isovitexin in cancer therapy: a critical review. Ann N Y Acad Sci. 1401(1):102-113.

Gao J., Morgan W.A., Sanchez-Medina A., Corcoran O. 2011. The ethanol extract of Scutellaria baicalensis and the active compounds induce cell cycle arrest and apoptosis including upregulation of p53 and Bax in human lung cancer cells. Toxicol Appl Pharmacol. 254(3):221-228

Hanafi M.M.M., Afzan A., Yaakob H., Aziz R., Sarmidi M.R., Wolfender J.L., et al. 2017. In vitro pro-apoptotic and anti-migratory effects of Ficus deltoidea L. plant extracts on the human prostate cancer cell lines PC3. Front Pharmacol. 8:895.

He M., Min J.W., Kong W.L., He X.H., Li J.X., Peng B.W. 2016. A review on the pharmacological effects of vitexin and isovitexin. Fitoterapia. 115:74-85.

Lee J.H., Chinnathambi A., Alharbi S.A., Shair O.H.M., Sethi G., Ahn K.S. 2019. Farnesol abrogates epithelial to mesenchymal transition process through regulating Akt/mTOR pathway. Pharmacol Res. 150:104504.

Li C.Y., Wang Q., Wang X., Li G., Shen S., Wei X. 2019. Scutellarin inhibits the invasive potential of malignant melanoma cells through the suppression epithelial-mesenchymal transition and angiogenesis via the PI3K/Akt/mTOR signaling pathway. Eur J Pharmacol. 858:172463.

Liang X., Xu C., Cao X., Wang W. 2019. Isovitexin suppresses cancer stemness property and induces apoptosis of osteosarcoma cells by disruption of the DNMT1/miR-34a/Bcl-2 axis. Cancer Manag Res. 11:8923-8936. 
Lin C.M., Huang S.T., Liang Y.C., Lin M.S., Shih C.M., Chang Y.C., et al. 2005. Isovitexin suppresses lipopolysaccharide-mediated inducible nitric oxide synthase through inhibition of NF-kappa B in mouse macrophages. Planta Med. 71(8):748-753.

Lv S.X. and Qiao X. 2018. Isovitexin (IV) induces apoptosis and autophagy in liver cancer cells through endoplasmic reticulum stress. Biochem Biophys Res Commun. 496(4):1047-1054.

McDermott F.T., Hughes E.S., Pihl E., Milne B.J., Price A.B. 1981. Prognosis in relation to symptom duration in colon cancer. Br J Surg. 68(12):846-849.

Mu D., Xiang H., Dong H., Wang D., Wang T. 2018. Isovitexin, a potential candidate inhibitor of sortase A of Staphylococcus aureus USA300. J Microbiol Biotechnol. 28(9):1426-1432.

Narayanankutty A. 2019. PI3K/ Akt/ mTOR Pathway as a therapeutic target for colorectal cancer: A review of preclinical and clinical evidence. Curr Drug Targets. 20(12):1217-1226.

Phipps E., Braitman L.E., Stites S., Leighton J.C. 2008. Quality of life and symptom attribution in long-term colon cancer survivors. J Eval Clin Pract. 14(2):254-258.

Rademaker G., Costanza B., Bellier J., Herfs M., Peiffer R., Agirman F., et al. 2019. Human colon cancer cells highly express myoferlin to maintain a fit mitochondrial network and escape p53-driven apoptosis. Oncogenesis. 8(3):21.

Sun V., Borneman T., Koczywas M., Cristea M., Piper B.F., Uman G., et al. 2012. Quality of life and barriers to symptom management in colon cancer. Eur J Oncol Nurs. 16(3):276-280.

Sun X., Ng T.T.H., Sham K.W.Y., Zhang L., Chan M.T.V., Wu W.K.K., et al. 2019. Bufalin, a Traditional Chinese Medicine compound, prevents tumor formation in two murine models of colorectal cancer. Cancer Prev Res (Phila). 12(10):653-666.

Wei R., Xiao Y., Song Y., Yuan H., Luo J., Xu W. 2019. FAT4 regulates the EMT and 
autophagy in colorectal cancer cells in part via the PI3K-AKT signaling axis. J Exp Clin Cancer Res. 38(1):112.

Yan S., Yue Y., Wang J., Li W., Sun M., Zeng L., et al. 2019. Banxia Xiexin decoction, a traditional Chinese medicine, alleviates colon cancer in nude mice. Ann Transl Med. 7(16):375.

Zhang J., Yuan K., Zhou W.L., Zhou J., Yang P. 2011. Studies on the active components and antioxidant activities of the extracts of Mimosa pudica Linn. from southern China. Pharmacogn Mag. 7(25):35-39.

Zhu M.L., Zhang P.M., Jiang M., Yu S.W., Wang L. 2020. Myricetin induces apoptosis and autophagy by inhibiting PI3K/Akt/mTOR signalling in human colon cancer cells. BMC Complement Med Ther. 20(1):209.

\section{Figure legends}

Figure 1 Isov inhibited colon cancer cells growth. $(\mathbf{n}=\mathbf{5})$ Magnification: $\times 200$. A: Isov inhibited cells growth in HCEC, HT29, SW480, HCT116, and LoVo cells. B: Colony formation assay was used to detect the proliferation of HCT116 cells. C: Colony formation assay was used to detect the proliferation of LoVo cells. D: Histograms show the colony numbers of HCT116 and LoVo cells. vs. control group, ${ }^{*} P<0.05,{ }^{* *} P<0.01$; vs. Isov group, ${ }^{\sharp} P<0.05$, ${ }^{\#} P<0.01$; vs. IGF-1 group, $\wedge P<0.05$.

Figure 2 Scratch wound healing assay and transwell assay were used to evaluate the effect of Isov on migration and invasion of colon cancer cells. $(\mathbf{n}=\mathbf{5})$ Magnification: $\times 200$, Scale bar: $200 \mu \mathrm{m}$. A: HCT116 cells with different treatment and representative images from scratch wound healing assays were acquired at 0 and $24 \mathrm{~h}$. B: Histograms show the wound closure rate of HCT116 cells. C: Lovo cells with different treatment and representative images from scratch 
wound healing assays were acquired at 0 and $24 \mathrm{~h}$. D: Histograms show the wound closure rate of LoVo cells. E: Transwell assay used to investigate HCT116 and LoVo cells invasion ability. F: Histograms show the number of invasion cells. vs. control group, ${ }^{*} P<0.05,{ }^{* *} P<0.01$; vs. Isov group, ${ }^{\#} P<0.05,{ }^{\#} P<0.01$; vs. IGF-1 group, ${ }^{\wedge} P<0.05$.

Figure 3 Isov induces colon cancer cells apoptosis. $(\mathbf{n}=\mathbf{5})$ A: HCT116 cells apoptosis was detected by flow cytometry. B: The apoptosis rate of HCT116 cells was quantified. C: LoVo cells apoptosis was detected by flow cytometry. D: The apoptosis rate of LoVo cells was quantified. E: The expression level of Bcl-2, Bax, and caspase-3 proteins were assessed by western blot. F: The expression level of Bcl-2 was quantified. G: The expression level of Bax was quantified. H: The expression level of caspase-3 was quantified. vs. control group, ${ }^{*} P<0.05$, ${ }^{* *} P<0.01$; vs. Isov group, ${ }^{\sharp} P<0.05,{ }^{\#} P<0.01$; vs. IGF-1 group, ${ }^{\wedge} P<0.05$.

Figure 4 Isov inhibited EMT in colon cancer cells. $(\mathbf{n}=\mathbf{3})$ A: The expression level of E-cadherin, N-cadherin, ZO-1, vimentin, slug, and snail proteins were assessed by western blot. The expression level of E-cadherin (B), N-cadherin (C), ZO-1 (D), vimentin (E), slug (F), and snail (G) proteins were quantified. vs. control group, ${ }^{*} P<0.05,{ }^{* *} P<0.01$; vs. Isov group, ${ }^{\#} P<$ $0.05,{ }^{\#} P<0.01$; vs. IGF-1 group, $\wedge P<0.05,{ }^{\wedge} \wedge P<0.01$.

Figure 5 Isov inhibited the expression levels of PI3K/Akt/mTOR in vitro. $(\mathrm{n}=5)$ A: The expression level of $\mathrm{p}-\mathrm{PI} 3 \mathrm{~K}, \mathrm{p}-\mathrm{Akt}$, and $\mathrm{p}-\mathrm{mTOR}$ proteins were assessed by western blot. B: The expression level of $\mathrm{p}-\mathrm{PI} 3 \mathrm{~K} / \mathrm{PI} \mathrm{K}$ was quantified. C: The expression level of $\mathrm{p}-\mathrm{Akt} / \mathrm{Akt}$ was quantified. D: The expression level of p-mTOR was quantified. vs. control group, ${ }^{*} P<0.05,{ }^{* *} P<$ 0.01 ; vs. Isov group, ${ }^{\#} P<0.05,{ }^{\#} P<0.01$; vs. IGF-1 group, $\wedge P<0.05, \wedge \wedge P<0.01$.

Figure 6 Isov suppressed colon cancer growth in vivo. (n=9) A: Image showing the inhibition 
410 of a xenografted colon tumor in nude mice treated with Isov. B: Tumor weights were recorded.

411 C: Tumor volumes were calculated. D: Tumor inhibition rate. vs. control group, ${ }^{*} P<0.05$.

Figure 7 Isov inhibited the expression levels of EMT markers in vivo. (n=9) A: The

413

414

415

416

417

418

419

420

421

422 expression level of E-cadherin, N-cadherin, ZO-1, vimentin, slug, and snail proteins were assessed by western blot, these were representative blots from single tumor. Histograms show the expression levels of E-cadherin (B), N-cadherin (C), ZO-1 (D), vimentin (E), slug (F), and snail (G) proteins were quantified. vs. control group, ${ }^{*} P<0.05,{ }^{* *} P<0.01$.

Figure 8 Isov inhibited the expression levels of proteins in vivo. (n=9) A: The expression level of PI3K, p-Akt, p-mTOR, Bcl-2, Bax, and caspase-3 proteins were assessed by western blot, these were representative blots from single tumor. B: Histograms show the expression levels of p-PI3K/PI3K (B), p-Akt/Akt (C), p-mTOR (D), Bcl-2 (E), Bax (F), and caspase-3 (G) proteins were quantified. All of the expression levels of protein were normalized to level of GAPDH. vs. control group, ${ }^{*} P<0.05,{ }^{* *} P<0.01$. 

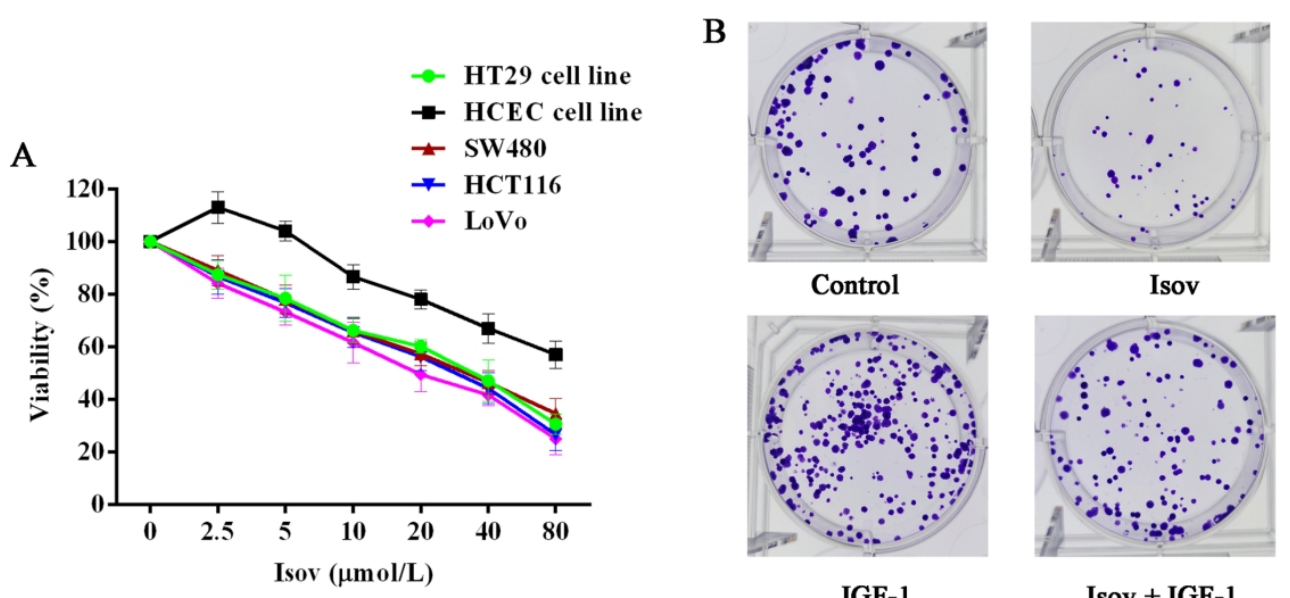

IGF-1

Isov + IGF-1
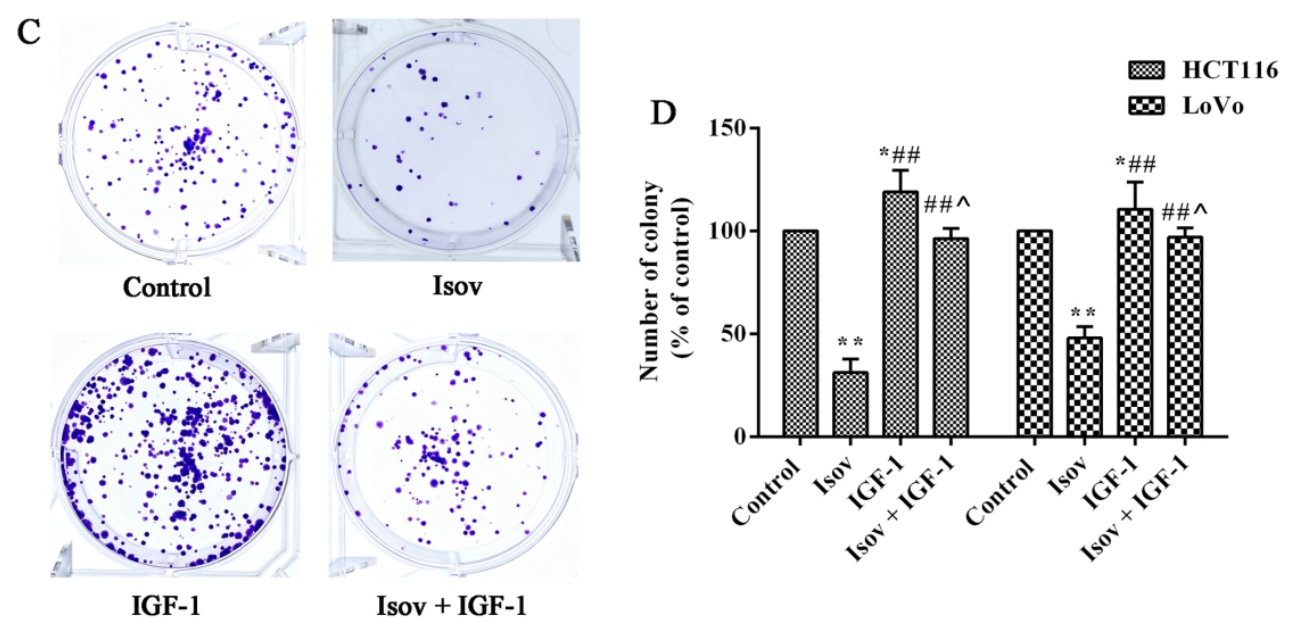

Isov inhibited colon cancer cells growth. 

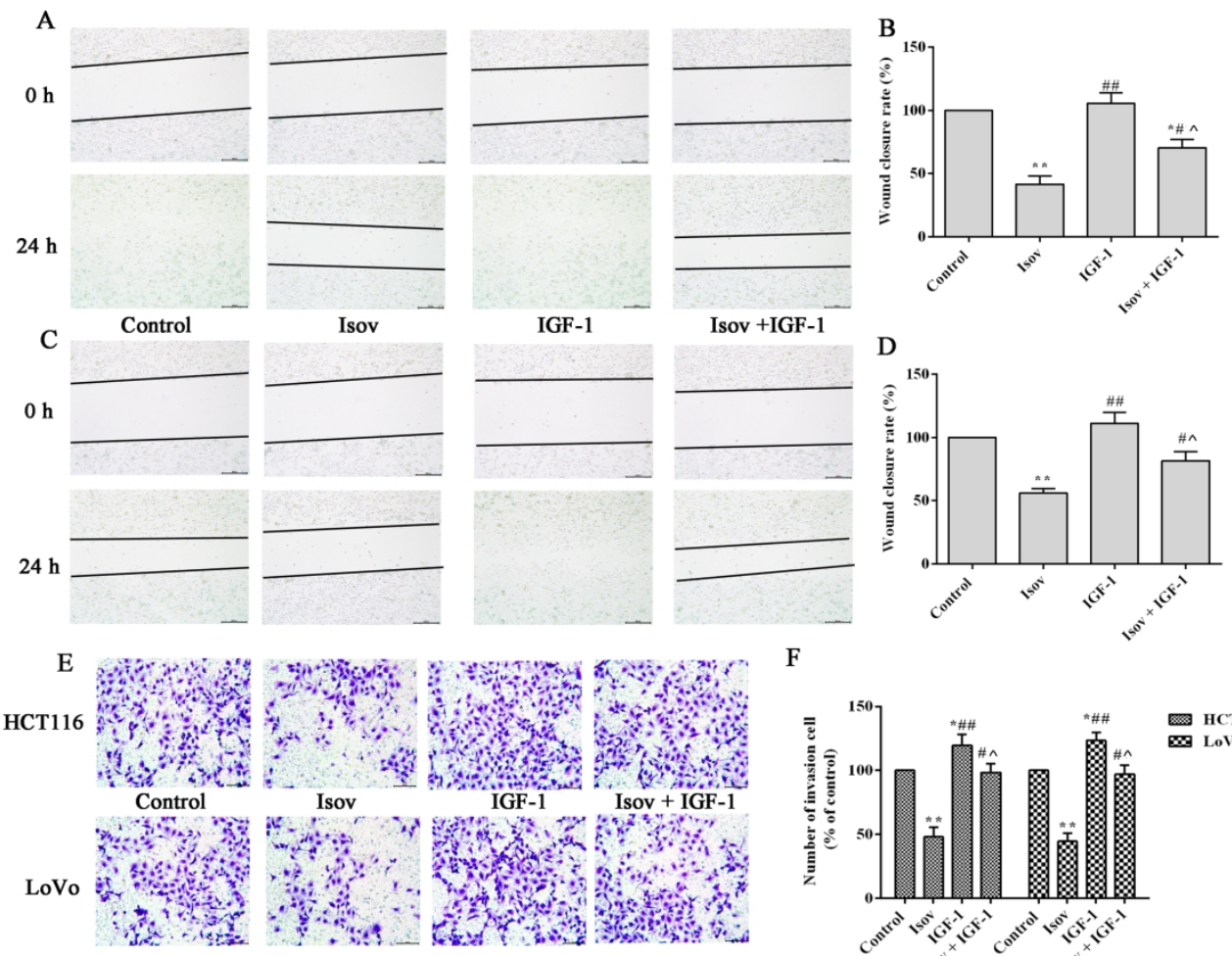

F

D
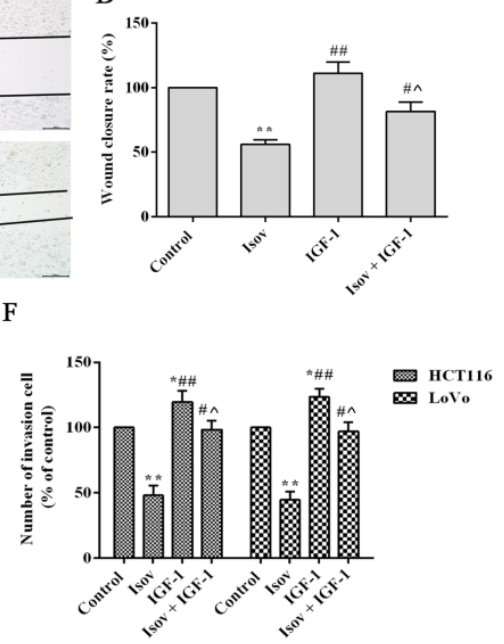

Figure 2 Scratch wound healing assay and transwell assay were used to evaluate the effect of Isov on migration and invasion of colon cancer cells. 

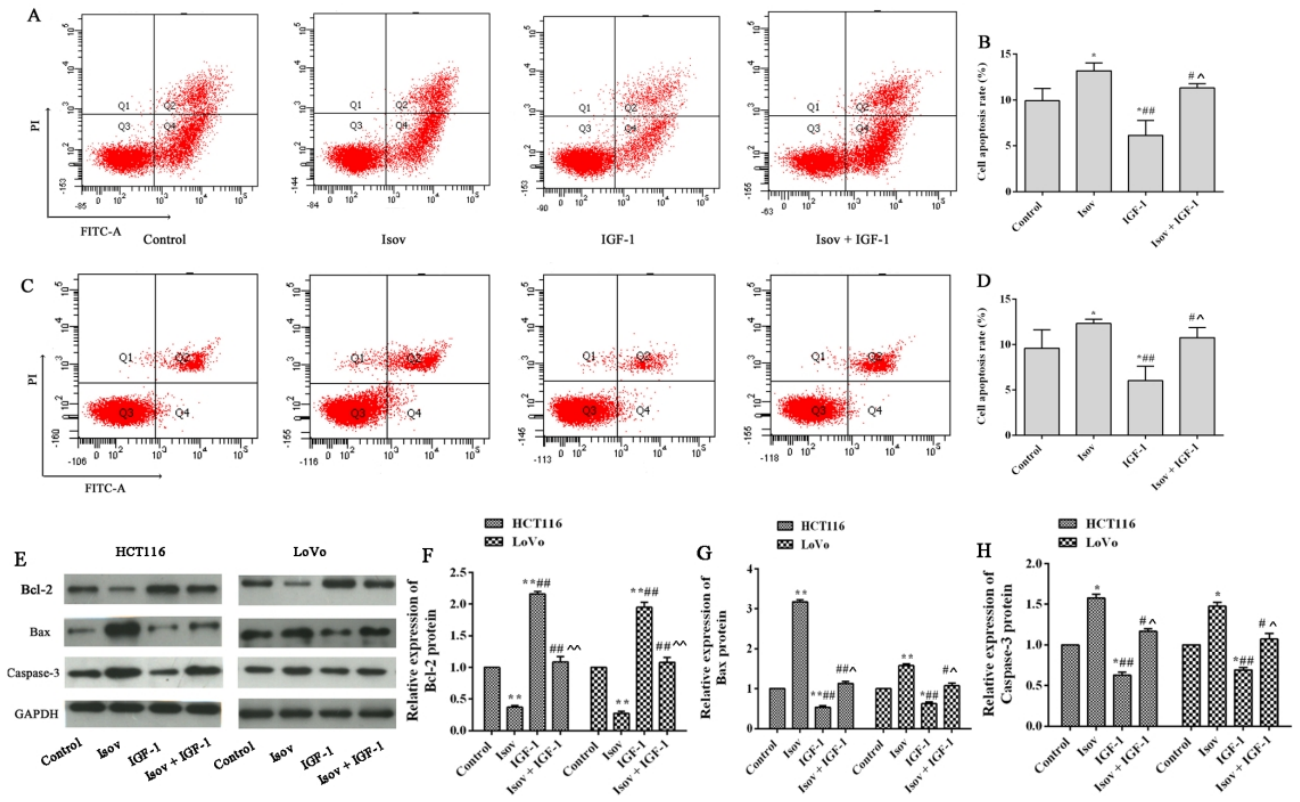

Figure 3 Isov induces colon cancer cells apoptosis. 

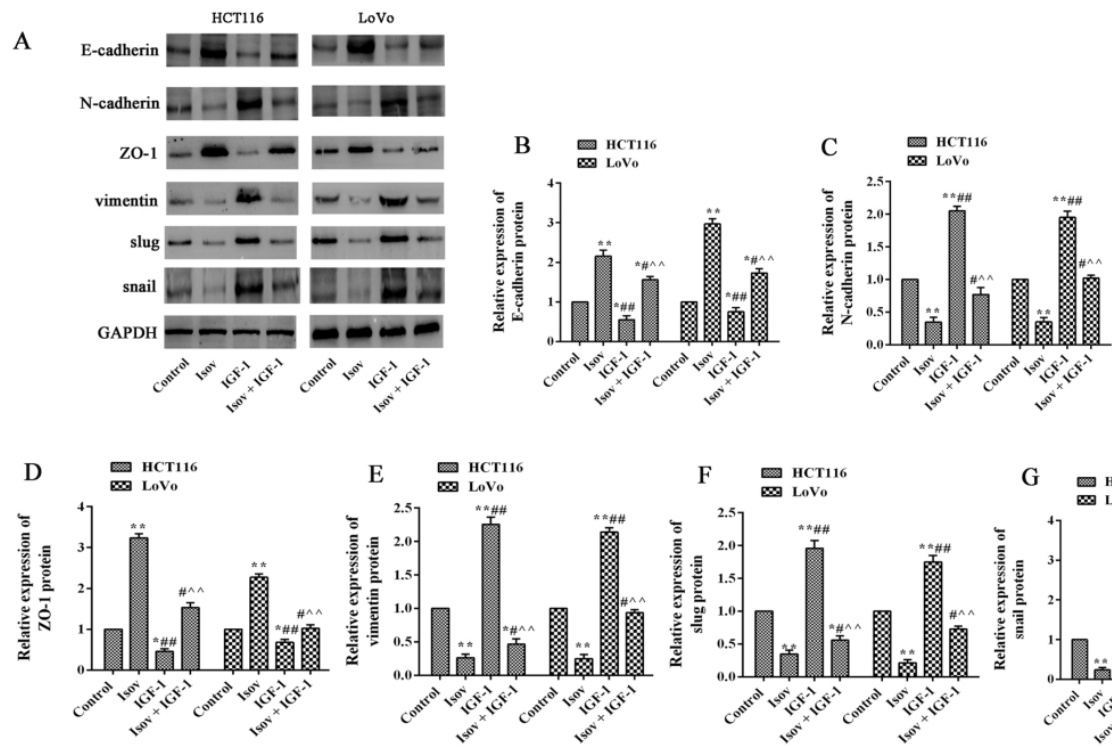

G 口 нстис

Figure 4 Isov inhibited EMT in colon cancer cells.

$180 \times 105 \mathrm{~mm}(300 \times 300 \mathrm{DPI})$ 

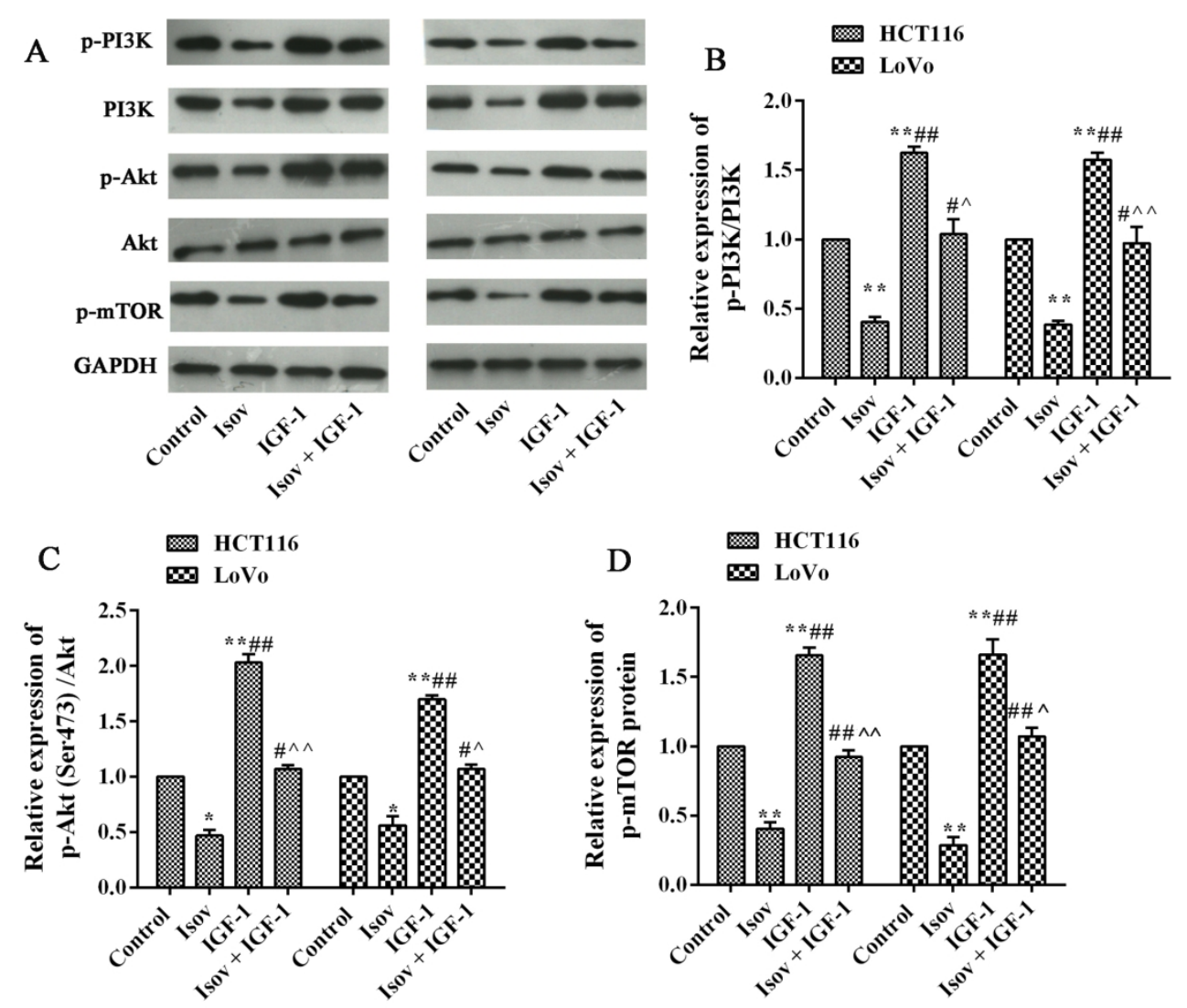

Figure 5 Isov inhibited the expression levels of PI3K/Akt/mTOR in vitro.

$180 \times 154 \mathrm{~mm}(300 \times 300$ DPI $)$ 
A
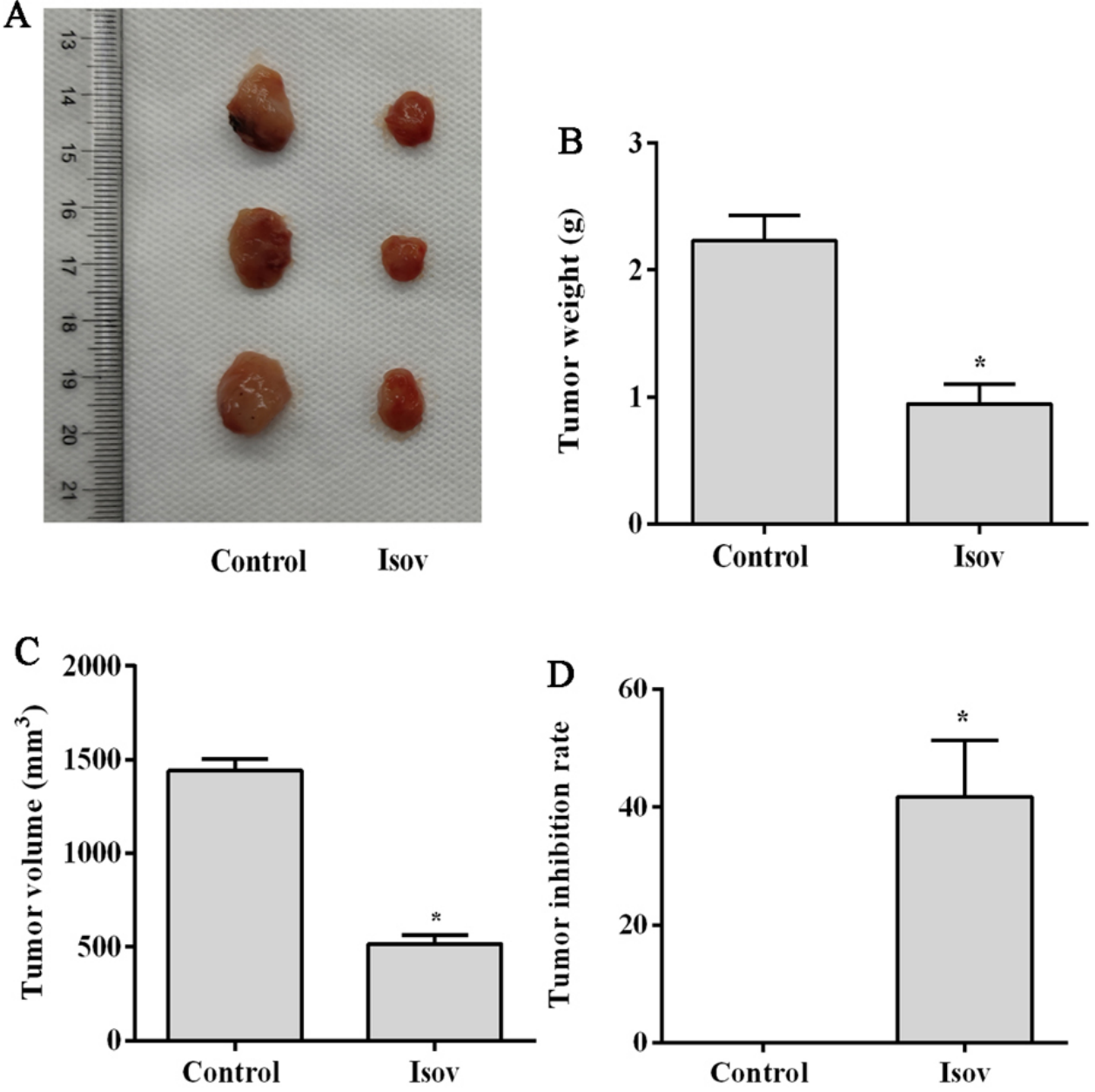

Figure 6 Isov suppressed colon cancer growth in vivo.

$80 \times 80 \mathrm{~mm}(300 \times 300 \mathrm{DPI})$ 

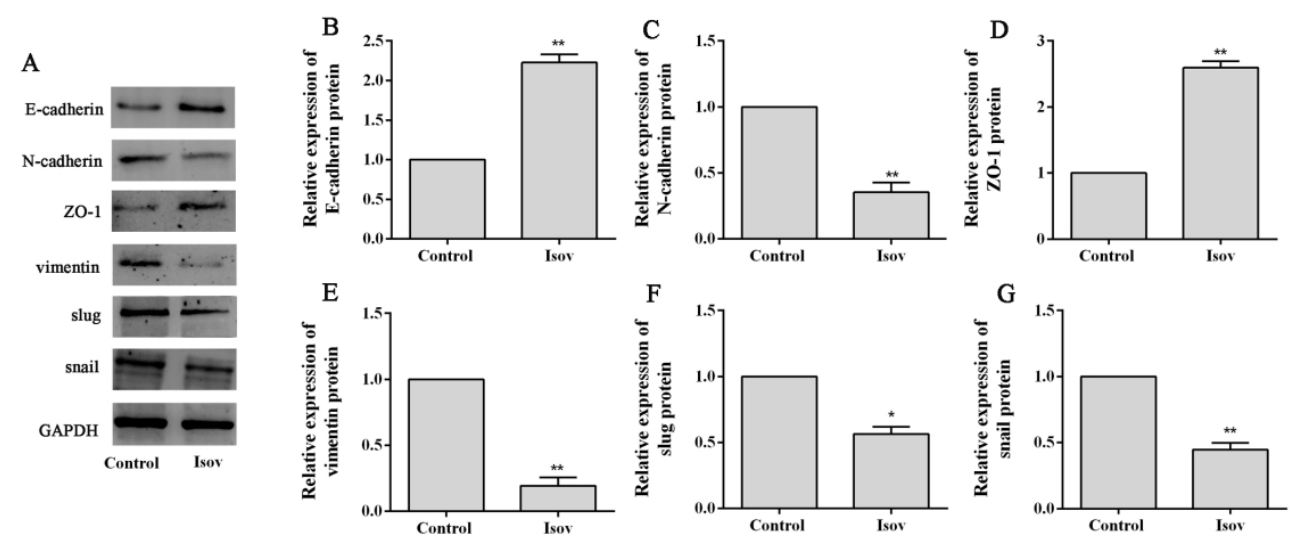

Figure 7 Isov inhibited the expression levels of EMT markers in vivo.

$180 \times 75 \mathrm{~mm}(300 \times 300 \mathrm{DPI})$ 

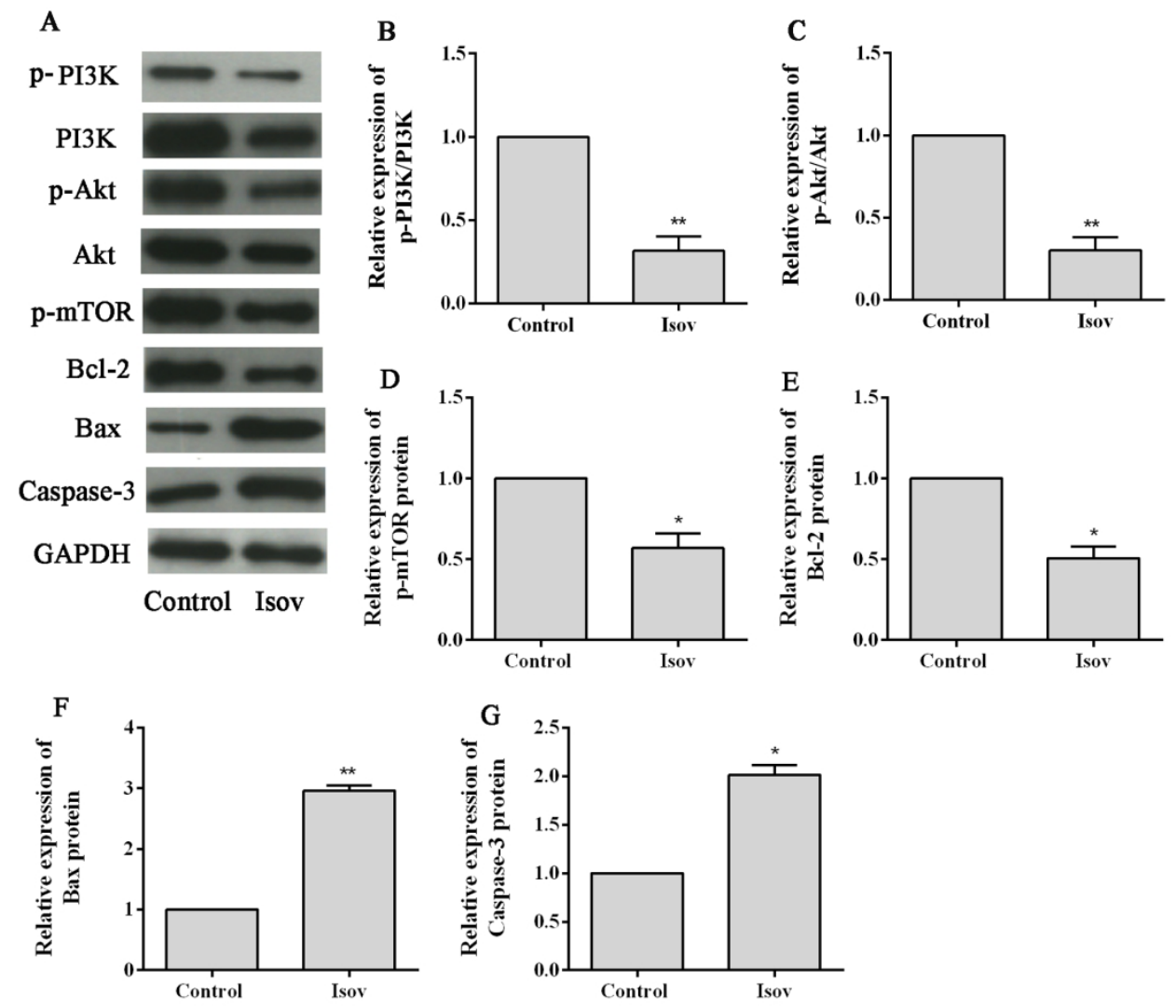

Figure 8 Isov inhibited the expression levels of proteins in vivo. 